\title{
Low-carbohydrate diets adversely impact the skin of a mouse model of photoaging exposed to ultraviolet $B$ radiation
}

\author{
Kazuma Kanaki, ${ }^{1}$ Yuko Otsuka, ${ }^{2}$ Rumi Hino, ${ }^{1,2}$ and Tomoko Kaburagi ${ }^{1,2, *}$ \\ ${ }^{1}$ Graduate School of Sports and Health Science and ${ }^{2}$ Department of Health Science, Daito Bunka University, \\ 560 Iwadono, Higashimatsuyama, Saitama 355-8681, Japan
}

(Received 3 February, 2021; Accepted 23 February, 2021)

\begin{abstract}
The study results regarding the effects of low-carbohydrate (LC) diets remain controversial; hence further research is required to assess their safety. Here, we examined whether LC diets cause skin damage in C57BL/6J mice. Six-week-old female mice $(n=20)$ were fed an LC (protein/fat/carbohydrate energy ratio $=35: 45: 20$ ) or control diet ad libitum for eight weeks, after which their backs were shaved, and a subset of the mice were exposed to ultraviolet B radiation thrice per week. Ultraviolet B irradiation induced wrinkle formation on the skin surface, and thickening of the epidermis, which was also noticeable in the LC diet-fed mice in the absence of ultraviolet $B$ radiation. Meanwhile, the number of epidermal melanocytes and degree of horny layer keratosis increased in the LC diet-fed mice following ultraviolet B irradiation. mRNA expression analysis of the liver and skin showed decreased levels of the antioxidant enzyme superoxide dismutase 1 following ultraviolet B irradiation only in the LC diet-fed mice. Alternatively, the expression of pro-inflammatory cytokines, tumor necrosis factor- $\alpha$ and interleukin-1 $\beta$, increased in response to ultraviolet B radiation and LC diet intake. Hence, LC diets may adversely affect skin morphology and exacerbate the effects of ultraviolet B irradiation, which may be associated with antioxidant dysfunction.
\end{abstract}

Key Words: low-carbohydrate diet, epidermal thickness, ultraviolet B, superoxide dismutase, pro-inflammatory cytokine

L ow-carbohydrate (LC) diets are widely recognized for their effects on the regulation of blood sugar and lipid levels and weight reduction. This diet has attracted attention as a means to treat, or even prevent, lifestyle-related diseases. ${ }^{(1-5)}$ However, different studies have reported varying effects elicited by an LC diet, with no clear consensus reached regarding the effects of LC diets on biological function. Notably, several of the reported positive effects of LC, including regulation of blood sugar and induction of weight loss, have only been observed in hyperglycemic or obese subjects, respectively. ${ }^{(1-5)}$ Meanwhile, results of our previous study demonstrated that LC diets had adverse effects on nonobese animal models, including kidney damage and lipid metabolism suppression, while having no effects on weight loss or blood sugar control. ${ }^{(6,7)}$ Moreover, the administration of LC diets has also been reported to accelerate aging and cause heart disease, hence raising questions about their safety. ${ }^{(8-12)}$ In recent years, many media outlets have promoted LC diets for the maintenance of good health (i.e., to prevent aging and obesity), causing a subsequent increase in the number of people following these diets, even among nonobese individuals. Meanwhile, a decrease in dietary carbohydrate levels has been found to be accompanied by an increase in dietary fat and protein levels. It is, therefore, necessary to examine the safety of LC diets as well as their potential impact on all aspects of human health.

The skin, considered to be the largest organ in humans, functions as a sensor for exogenous stimuli, undergoing dynamic changes in response to internal and external environmental changes. ${ }^{(13-15)}$ Okouchi et al. ${ }^{(11)}$ reported that LC diets aggravate skin aging in SAMP8 mice, a model of accelerated aging. Meanwhile, photoaging, which is distinct from chronological aging, is defined as deterioration of the skin morphology as a result of ultraviolet (UV) radiation. ${ }^{(13,14,16)}$ Both chronological aging and photoaging induce similar changes in fundamental molecular pathways. ${ }^{(17)}$ Specifically, abnormal keratinization and inflammatory reactions occur as a result of skin exposure to UV radiation. While chronic UV exposure causes photoaging, and adversely affects the skin morphology by promoting the formation of senile pigment spots and wrinkles, as well as sagging and dryness, which are known to be induced by oxidative stress. ${ }^{(17-19)}$ UV radiation is comprised of $5 \%$ ultraviolet $B$ (UVB), which is primarily absorbed by the epidermis, the outer layer of the skin. This type of UV radiation is considered very powerful, damaging cells and causing skin inflammation and sunburns..$^{(13,15,16,19)}$ Although LC diets are presumed to have an effect on photoaging, their effects when combined with UV radiation, remains unknown.

Hence, the current study used a rodent model to investigate the effect of a LC diet with a moderately-high fat and protein composition. Moreover, the mice were irradiated with UVB following LC or normal diet intake and examined for changes in skin morphology as well as in oxidative stress and pro-inflammatory cytokine expression within the skin.

\section{Materials and Methods}

Animals and diet. Six-week-old specific pathogen-free female C57BL/6J mice $(n=20$; Sankyo Lab Services, Inc., Tokyo, Japan) were randomly housed in cages (five animals per cage) in a temperature-controlled room at $24^{\circ} \mathrm{C}$ with a $12 \mathrm{~h}$ light/ dark cycle. The number of mice in each group was set to five as the lowest number that could be statistically analyzed. All mice were initially fed a control diet (AIN-93G diet) for one week during the acclimation period. They were then divided into two groups, an LC group [protein/fat/carbohydrate (PFC) energy ratio $=45: 35: 20]$ and a control group $(\mathrm{PFC}$ energy ratio $=20: 16: 64)$ and were maintained for eight weeks. Table 1 shows the compo-

*To whom correspondence should be addressed.

E-mail: t-kabu@ic.daito.ac.jp 
Table 1. Composition of the experimental diet

\begin{tabular}{|c|c|c|}
\hline & Control & LC \\
\hline \multicolumn{3}{|l|}{ PFC energy ratio (\%) } \\
\hline Carbohydrate & 64 & 20 \\
\hline Protein & 20 & 35 \\
\hline Fat & 16 & 45 \\
\hline Energy (kcal/g) & 4.0 & 4.8 \\
\hline$(\mathrm{kJ} / \mathrm{g})$ & 16.7 & 20.1 \\
\hline \multicolumn{3}{|l|}{ Ingredient (g/kg) } \\
\hline Casein & 200.0 & 345.0 \\
\hline Cornstarch & 397.5 & - \\
\hline Maltodextrin 10 & 132.0 & 132.0 \\
\hline Sucrose & 100.0 & 64.9 \\
\hline cellulose & 50.0 & 50.0 \\
\hline Soybean oil & 70.0 & 70.0 \\
\hline Lard & - & 130.0 \\
\hline Mineral mix S10022 $\mathrm{G}^{+}$ & 35.0 & 0.0 \\
\hline Mineral mix S10022 $C^{\ddagger}$ & - & 3.5 \\
\hline Calcium carbonate & - & 12.5 \\
\hline Potassium citrate & - & 6.5 \\
\hline Potassium phosphate & - & 1.8 \\
\hline Sodium chloride & - & 2.6 \\
\hline Vitamin mix V10037§ & 10.0 & 10.0 \\
\hline L-Cystine & 3.0 & 5.2 \\
\hline Choline bitartrate & 2.5 & 2.5 \\
\hline$t$-Butylthdroquinone & 0.014 & 0.014 \\
\hline
\end{tabular}

A control and LC diet were prepared using material composition based on AIN-93G (American Institute of Nutrition). Control, control diet; LC, low-carbohydrate diet; PFC, protein/fat/carbohydrate. ${ }^{\dagger} \mathrm{AIN}-93 \mathrm{G}$ mineral mix, ${ }^{\ddagger}$ AIN-93G mineral mix except for calcium carbonate, potassium citrate, sodium chloride (Research Diet Inc.), ${ }^{5}$ Vitamin Mix for AIN-93 with Biotin (including vitamin A, vitamin D3, vitamin $E$, and vitamin $B$ group, Research Diet Inc.)

sition of the experimental diet. The mineral content, including the calcium, potassium, phosphorus, and sodium levels were adjusted to be equal in the two experimental diets. Mice were allowed free access to food and drinking water throughout the acclimation and experimental periods. Individual mouse weights and food consumption rates per cage were recorded twice weekly. At the end of 8 -week feeding period, the mice were fasted for $8 \mathrm{~h}$ then sacrificed cervical dislocation under anesthesia by isoflurane inhalation. This study was approved by the Daito Bunka University Animal Experiment Committee (ASH18-006) and complied with the guidelines of the Japanese Council on Animal Research at Daito Bunka University in Saitama, Japan.

UVB irradiation. Irradiation was performed using a GL20SE lamp (Sankyo Denki Co., Ltd., Tokyo, Japan) as the UVB irradiation source. All mice were allowed to move freely within the cage, with the distance between the lamp and the back of the mouse set at $30 \mathrm{~cm}$, and a dose of $120 \mathrm{~mJ} / \mathrm{cm}^{3}$ applied as the minimum erythema dose. ${ }^{(15)}$ This procedure was carried out once for $1 \mathrm{~h}$ per day, three days per week for a total of eight weeks. This dose was based on that described in a previous study, to match the typical 2-h doses received by humans following exposure to UV rays in Osaka, Japan. ${ }^{(15)}$ Notably, the UVB lamp used in this experiment (i.e., GL20SE) is confirmed, from its absorption spectrum, to contain no ultraviolet C (less than $280 \mathrm{~nm}$ ).

Serum and tissue sampling. At the end of 8-week feeding period, the mice were fasted for $8 \mathrm{~h}$ then anesthetized by isoflurane inhalation. Blood was sampled through the orbital cavity and separated to serum by centrifugation. They were then sacrificed by cervical dislocation under anesthesia, and the livers, kidneys, spleen, and the left and right perirenal fat were immediately removed and weighed.

Morphological observation of the skin. After dissection, a portion of the dorsal skin at the UVB irradiation site (area: $1 \times$ $2 \mathrm{~cm}$ ) was collected, fixed with formalin, embedded in paraffin, and sliced into $3-\mu \mathrm{m}$-thick sections, which were then stained with hematoxylin and eosin (H\&E). Epidermal thickness was measured at 20 random points per mouse, excluding the upper portion of the pore and sweat glands. Epidermal thickness and subcutaneous fat levels were measured using ImageJ software (ver. 1.51, National Institutes of Health, Bethesda, MD).

Histological analysis of perirenal fat. The perirenal fat were then fixed in formalin, embedded in paraffin, sliced into 3-mm-thick sections, and stained with H\&E. The adipocyte area was measured at 50 random points per mouse and analyzed using ImageJ.

Gene expression analysis using real-time PCR. Hepatic and skin RNA extraction was performed using Isogen II (Nippon Gene Co., Ltd., Toyama, Japan), according to the manufacturer's instructions. Subsequently, $1 \mu \mathrm{g}$ of total RNA was reverse transcribed using a PrimeScript RT Reagent Kit (Takara Bio Inc., Shiga, Japan). Real-time PCR was performed using SYBR Green Master Mix (Takara Bio Inc.) and gene-specific primers (Applied Biosystems, Foster City, CA). Table 2 shows the primer sequences used for the amplification of Sod1, Gpx1, Foxol, Tnfa, and Illb. Relative changes in the expression of each gene were analyzed and normalized using the $18 \mathrm{~S}$ ribosomal RNA expression as an internal standard.

Statistical analysis. All results are presented as the average value \pm SE. All statistical analyses were performed using SPSS Statistics ver. 19.0 (IBM Corp., Armonk, NY), with the significance level set to $<5 \%$. After normality was confirmed using Shapiro-Wilk normality test, two-way analysis of variance (ANOVA) was performed. Multiple comparison tests between groups were performed using Tukey's method for items for which an interaction was observed.

\section{Results}

Changes in body weight, organ weight, and energy intake. During the experimental period, the body weight of mice fed the LC diet was significantly higher than that of the mice fed the control diet (Fig. 1, Table 3; LC diet effect: $p=0.041$ ). Liver weight was found to significantly increase following UVB irradiation (Table 3; UVB effect: $p<0.05$ ). Kidney and spleen weights

Table 2. Primers used for real-time PCR

\begin{tabular}{lccr}
\hline \multicolumn{1}{c}{ Gene } & Abbr. & Forward primer 5'-3' (F) & Reverse primer 5'-3' (R) \\
\hline Superoxide dismutase 1 & Sod1 & ACCAGTGCAGGACCTCATTTTAA & AGGTCTCCAACATGCCTCTCTTC \\
Glutathione peroxidase 1 & Gpx1 & CCTTGCCAACACCCAGTGA & GGCACACCGGAGACCAAAT \\
Forkhead box 01 & Foxo1 & ACGGGCTGTCTGTCTGTC & TAAGTGAAGTTTCTCTGTGGTTTC \\
Tumor necrosis factor-alpha & Tnfa & GGTGCCTATGTCTCAGCCTCTT & GCCATAGAACTGATGAGAGGGAG \\
Interleukin-1 beta & IL1b & CAACCAACAAGTGATATTCTCCATG & AGATCCACACTCTCAGCTGCA \\
\hline
\end{tabular}




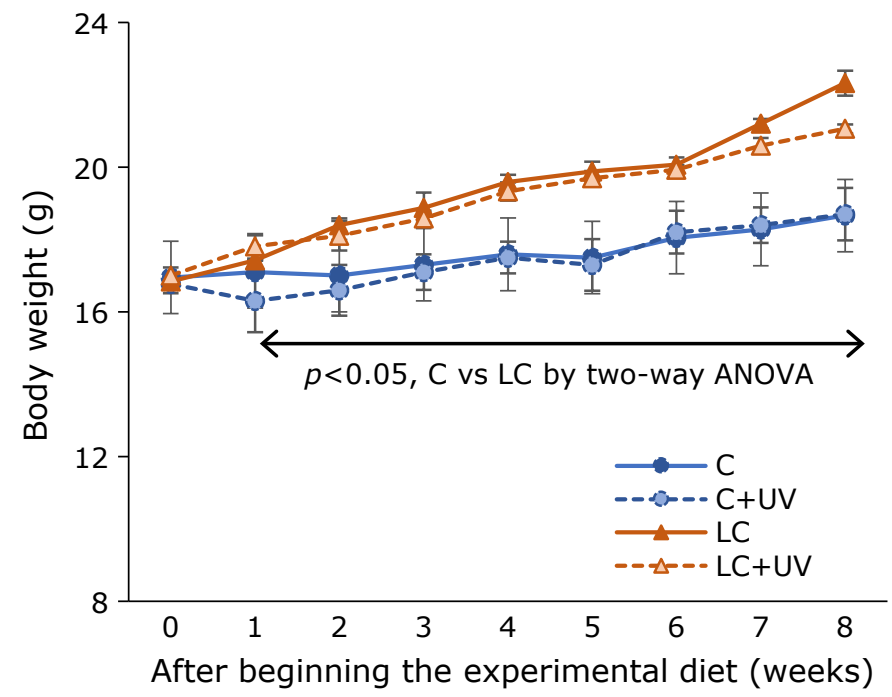

Fig. 1. Body weight changes. Both control and LC diets were provided ad libitum for eight weeks, and the body weight was measured twice a week. C, control diet; C + UV, control + UVB irradiation; LC, lowcarbohydrate diet; LC + UV, low-carbohydrate diet + UVB irradiation. The values represent the mean \pm SE $(n=5)$.

significantly increased in the mice fed the LC diet (Table 3; LC diet effect: $p<0.05)$. As five mice were housed per cage, the experimental diet intake per animal was measured by dividing the whole intake by the number of mice in each cage. However, no difference was observed in the energy intake (Table 3). In addition, no difference in fasting blood glucose was observed between the groups (Table 4).

Morphological observation of the skin. Morphological examination showed that UVB radiation caused the formation of wrinkles in mice fed the LC diet and those fed the control diet, with deeper wrinkles and pigmentation observed in mice fed the LC diet (Fig. 2A). Morphological observation confirmed that the UVB radiation also caused significant thickening of the epidermis (Fig. 2B; indicated by the black arrow, Fig. 2D; UVB effect, $p=0.001)$. In addition, epidermal thickening was noted in the mice fed the LC diet (Fig. 2D; LC diet effect; $p=0.023$ ). In comparison to those fed the control diet, mice fed the LC diet exhibited an increase in the number of melanocytes (Fig. 2B indicated by the white arrows) and parakeratosis cells (Fig. 2B; indicated by the red arrows), as well as the degree of keratinization in the keratin layer under UVB irradiation (Fig. 2B; indicated by the green arrows). Parakeratosis cells were observed in all mice fed the LC diet exposed to UVB radiation (Fig. 2B; indicated by the red arrows), however, were not seen in the mice from any other group. We also observed that the area of subcutaneous adipocytes significantly decreased after exposure to UVB radiation (Fig. 2C and E; UVB effect: $p<0.001$ ), while the LC diet had no effect on the subcutaneous adipocyte area (Fig. 2C).

Perirenal fat weight and adipocyte area. We observed that the perirenal adipocyte area was significantly enlarged in mice fed the LC diet (Fig. 3A and C; LC diet effect: $p=0.010$ ), however, was significantly decreased in size after exposure to UVB radiation (Fig. 3A and C; UVB effect: $p=0.002$ ). In addition, the perirenal fat tissue weight significantly increased after mice were fed the LC diet (Fig. 3B; LC diet effect: $p=0.014$ ), and significantly decreased following exposure to UVB radiation (Fig. 3B; UVB effect: $p=0.025$ ).

Hepatic and skin mRNA expression levels of antioxidant and antioxidant-related factors. The mRNA expression levels of antioxidant and antioxidant-related factors in the liver and skin were measured. Analysis using two-way ANOVA showed an interaction between the effects of LC diet and UVB on the liver and skin expression of Sod1 (Fig. 4A; $p=0.029$, Fig. 4D; $p=0.018)$. We subsequently performed a multiple comparison test for each group and found that the hepatic and skin Sod1 expression levels significantly decreased after exposure to UVB radiation only in mice fed the LC diet (Fig. 4A: $p=0.030$, Fig. 4D: $p=0.044$ ). Moreover, the Gpxl and Foxol expression levels showed a decreasing tendency after exposure to UVB radiation in the liver and skin; however, no significant difference was observed (Fig. 4B, C, E, and F). Gene expression analysis in the liver and skin showed that both the LC diet and UVB irradiation had similar effects.

Skin mRNA expression levels of pro-inflammatory cytokines. We observed that the mRNA expression levels of Tnfa and $I l l b$ significantly increased in the skin of mice following exposure to UVB radiation (Fig. 5A and B; UVB effect: $p<0.001)$. These pro-inflammatory cytokines were also

Table 3. Body weight, organs weight, and food intake

\begin{tabular}{lcccc}
\hline & Control & LC & Control + UVB & LC + UVB \\
\hline Initial body weight $(g)$ & $15.52 \pm 0.11$ & $15.26 \pm 0.11$ & $15.53 \pm 0.21$ & $15.78 \pm 0.14$ \\
Final body weight $(\mathrm{g})$ & $18.71 \pm 0.31$ & $21.97 \pm 0.22$ & $18.51 \pm 0.20$ & $21.4 \pm 0.20$ \\
Weight gains $(\mathrm{g})$ & $3.19 \pm 0.09$ & $6.71 \pm 0.05$ & $2.98 \pm 0.09$ & $5.62 \pm 0.06$ \\
${\text { liver }(\mathrm{g})^{\dagger}}_{\text {kidney }(\mathrm{g})^{\dagger}}$ & $0.672 \pm 0.028$ & $0.781 \pm 0.044$ & $0.778 \pm 0.024$ & $0.849 \pm 0.032$ \\
spleen $(\mathrm{g})^{\dagger}$ & $0.211 \pm 0.01$ & $0.279 \pm 0.008$ & $0.226 \pm 0.01$ & $0.267 \pm 0.013$ \\
perirenal fat $(\mathrm{g})^{\dagger}$ & $0.145 \pm 0.025$ & $0.183 \pm 0.01$ & $0.154 \pm 0.019$ & $0.175 \pm 0.029$ \\
food intake $(\mathrm{g} /$ day/mice $)$ & $0.145 \pm 0.027$ & $0.323 \pm 0.02$ & $0.126 \pm 0.048$ & $0.237 \pm 0.031$ \\
\hline
\end{tabular}

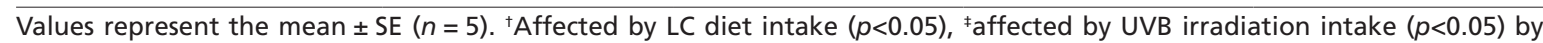
Two-way ANOVA.

Table 4. Fasting blood glucose concentration

\begin{tabular}{lcccc}
\hline & Control & LC & Control + UVB & LC + UVB \\
\hline Glucose $(\mathrm{mg} / \mathrm{dl})$ & $168.1 \pm 24.3$ & $173.7 \pm 23.9$ & $160.3 \pm 26.8$ & $176.5 \pm 23.9$ \\
\hline Values represent the mean \pm SE $(n=5)$. & & &
\end{tabular}



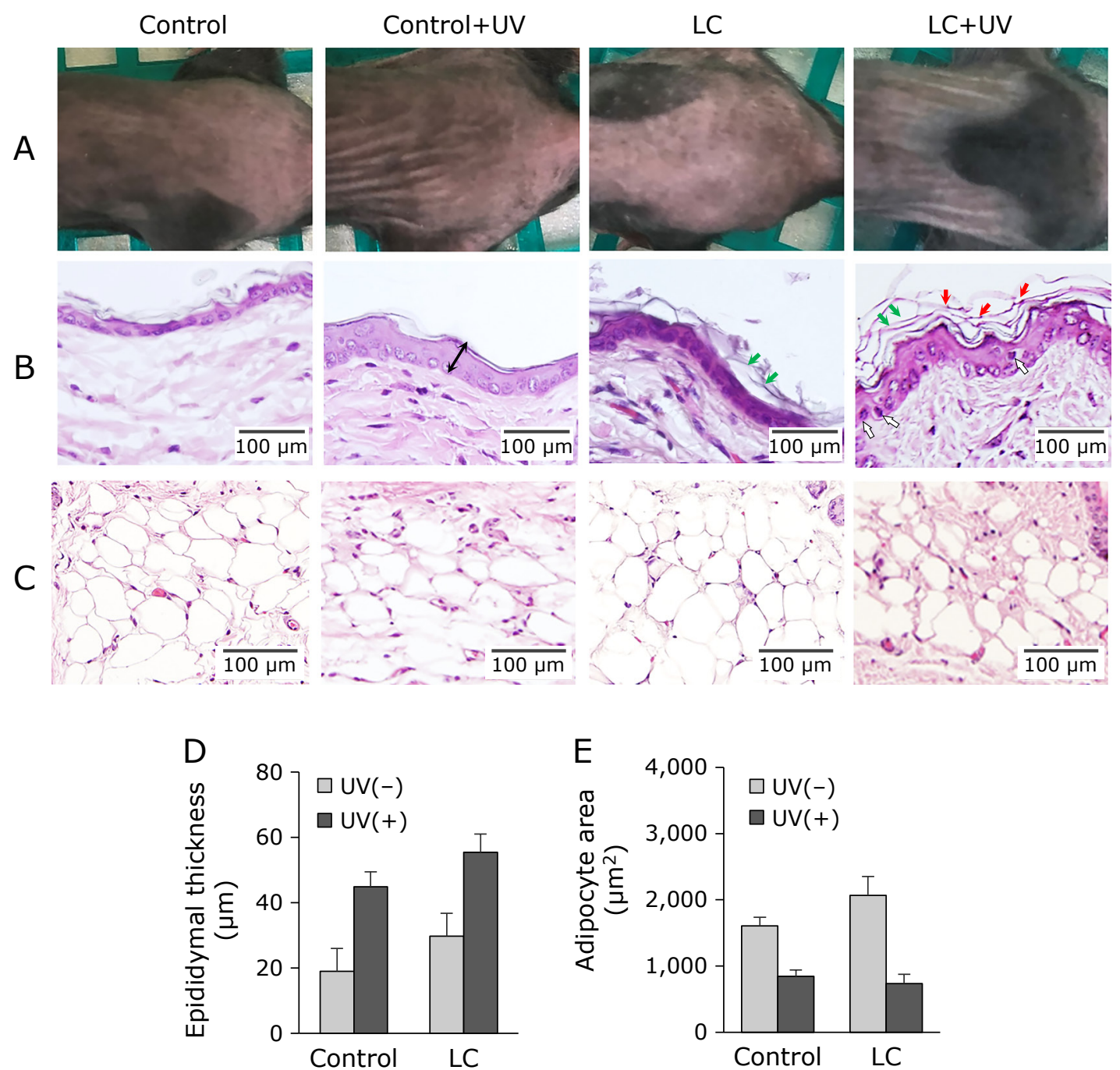

Fig. 2. Histological observation of the skin and wrinkle pigmentation on the back of a mouse exposed to UVB radiation. (A) Macroscopic findings of the back of a mouse. (B) H\&E-stained images of the skin. Black arrows indicate the epidermal thickness, green arrows indicate keratinization of the keratin layer, red arrows indicate parakeratosis, white arrows indicate melanocytes. (C) H\&E-stained image of subcutaneous fat. (D) Epidermal thickness. (E) Subcutaneous adipocyte area. Values are mean \pm SE $(n=5)$. The results of the two-way ANOVA were as follows: (D) LC diet: $p=0.023$, UVB irradiation: $p=0.001$; (E) UVB irradiation: $p<0.001$. The values represent the mean $\pm \operatorname{SE}(n=5)$. See color figure in the on-line version.

significantly upregulated in the skin of mice fed the LC diet (Fig. 5A, LC diet effect: $p=0.048$; Fig. 5B, LC diet effect: $p=0.009)$.

\section{Discussion}

Herein, we have demonstrated, for the first time, that an LC diet adversely impacted mouse skin morphology, which was even more pronounced during photoaging triggered by application of UVB radiation. This phenomenon was suggested to be associated with a decrease in Sod1 gene expression in the skin and liver due to an LC diet and UVB irradiation.

Thickening of the epidermis is one of the most prominent signs of photoaging, which is considered to be an extrinsic type of aging caused by UV rays and is a phenomenon not observed during endogenous aging.(11,14,17,18) Chronological skin aging causes the skin to atrophy and become thin, whereas photoaging causes the epidermis to become thicker as a defense mechanism to protect the basal cell layer and dermal layer from UV rays. UVB radiation generates reactive oxygen species (ROS) in the skin tissue, inducing the production of pro-inflammatory cytokines (TNF- $\alpha$, IL-1 $\beta$, etc.), activation of transcription factor activator protein-1 (AP-1) and nuclear factor- $\kappa \mathrm{B}(\mathrm{NF}-\kappa \mathrm{B})$, and increasing the production of matrix metalloproteinases (MMPs). ${ }^{(14-18)}$ In particular, the expression of MMP-1, MMP-3, and MMP-9 in skin fibroblasts, which induce epidermis thickening, are known to be upregulated by UVB radiation. ${ }^{(20)}$ These effects are mediated by the activation of stress kinase c-jun $\mathrm{N}$-terminal kinase 2 via generation of ROS and lipid peroxidation by the ion-driven Fenton reaction. ${ }^{(10)}$ Therefore, to eliminate the effects of ROS generated by UVB, antioxidants, such as superoxide dismutase (SOD), glutathione peroxidase (Gpx), catalase, and forkhead box O1 (Foxo1) are expressed in the skin tissue.

Our results further revealed that thickening of the epidermis in groups fed an LC diet with or without exposure to UV radiation. Although epidermal thickening was significantly induced by UVB radiation, this effect was most pronounced in LC-fed mice. This can be explained by the reduced antioxidant capacity due to reduced Sod1 levels in the liver and skin. Although no difference was observed statistically in the mRNA levels of Gpxl and Foxol, they tended to decrease in both the control and the LC diet groups. 

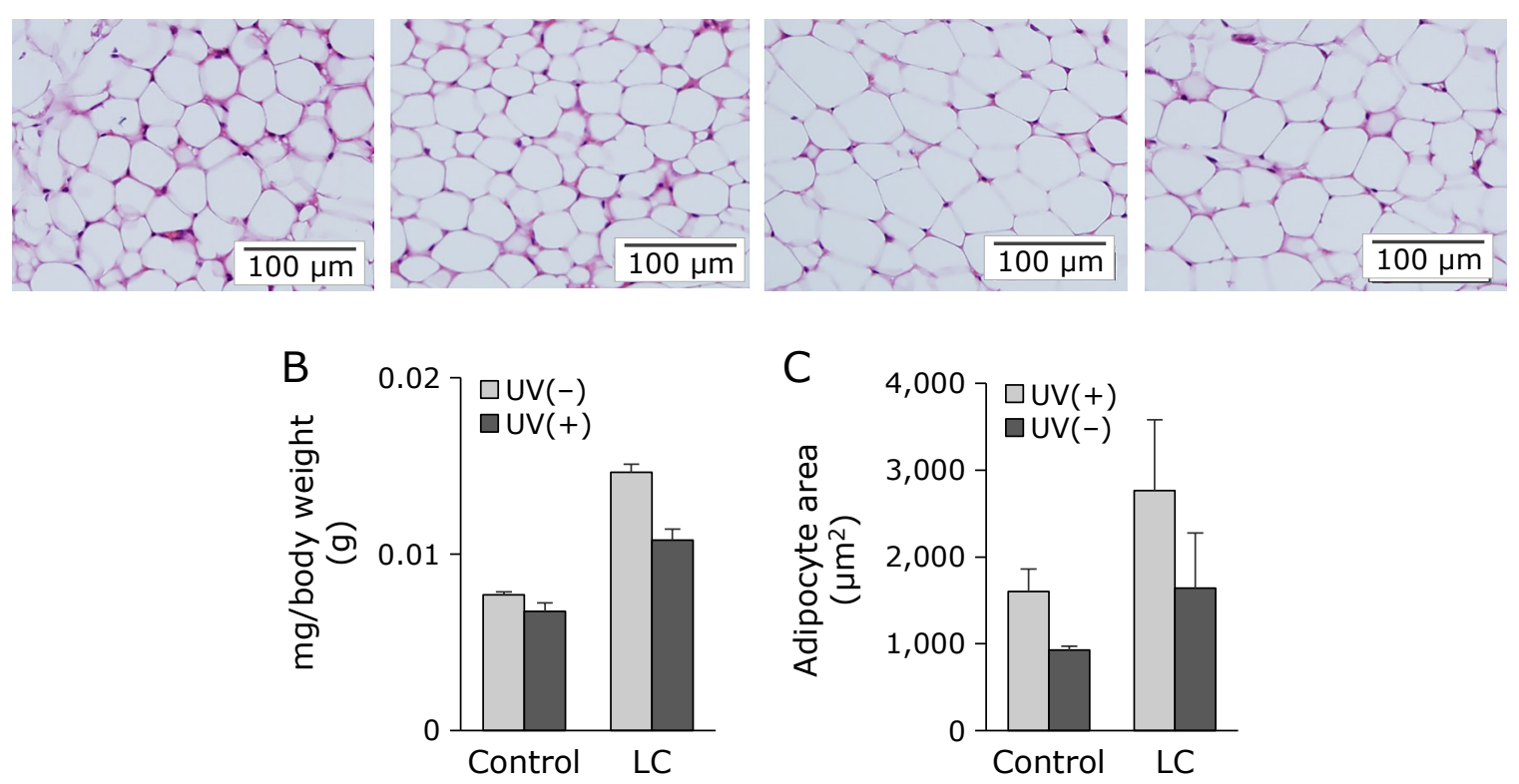

Fig. 3. Weight and histological observation of perirenal adipose tissue. (A) H\&E staining. (B) Adipose tissue weight. (C) Adipocyte area. The results of the two-way ANOVA were as follows: (B) LC diet: $p=0.014$, UVB irradiation: $p=0.025$; (C) LC diet: $p=0.010$, UVB irradiation: $p=0.002$. The values represent the mean \pm SE $(n=5)$.
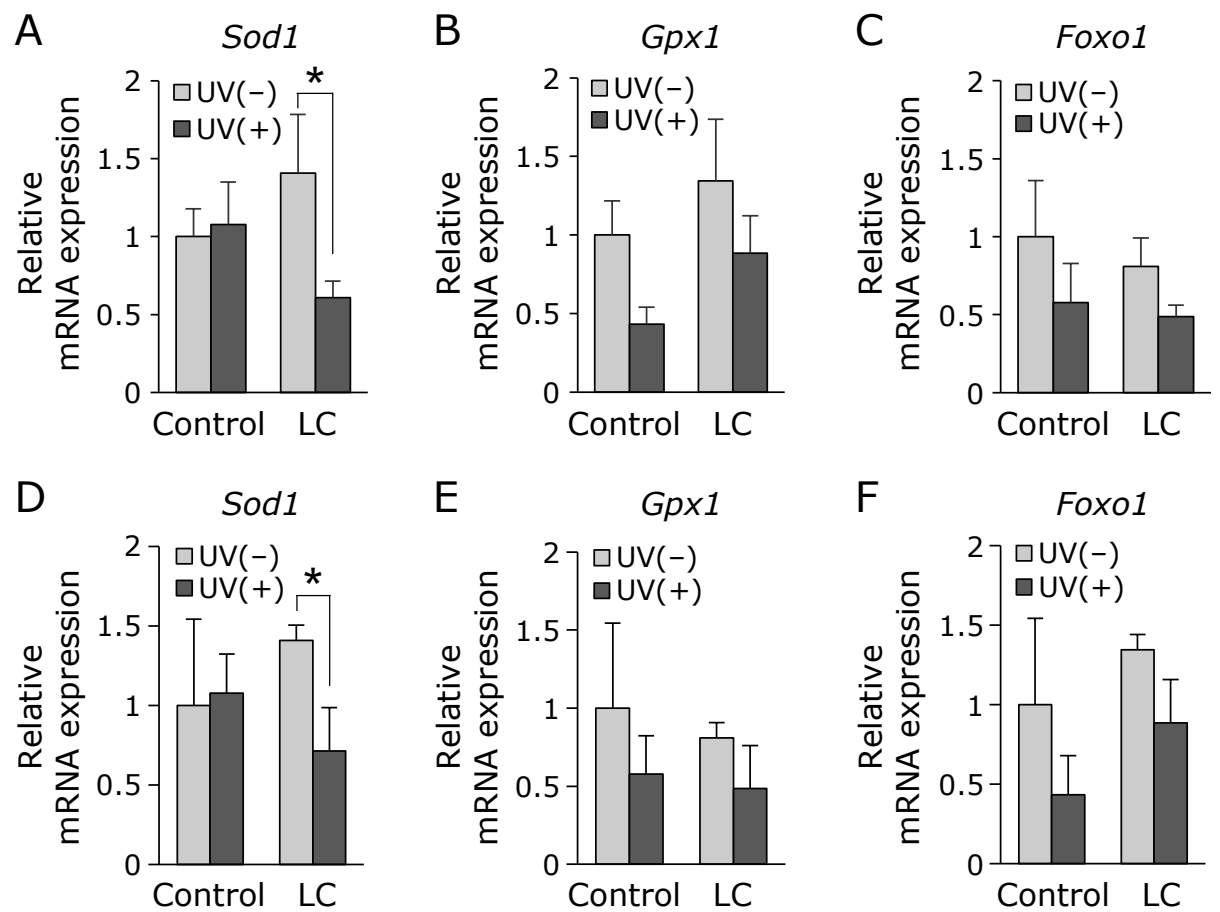

Fig. 4. Hepatic and skin mRNA levels of antioxidant and antioxidant-related factors. Hepatic mRNA expression normalized to the levels of $18 \mathrm{~S}$ ribosomal RNA as a loading control. The results of the two-way ANOVA were as follows: (A) LC diet $\times$ UVB irradiation: $p=0.030$; (D) LC diet $\times$ UVB irradiation: $p=0.044 ;{ }^{*} p<0.05$ by Tukey's post hoc test. The values represent the mean \pm SE $(n=5)$.

Additionally, the mRNA expression of pro-inflammatory cytokines, Tnfa and $I l l b$, was markedly upregulated in the skin of mice following UVB irradiation, as well as following ingestion of the LC diet. Therefore, it was speculated that the LC diet caused thickening of the skin epidermis by increasing proinflammatory cytokines in response to decreased antioxidants, including not measured in this experiment. Moreover, the presence of parakeratosis and melanocytes in the UVB radiated LC diet group also implies the action of ROS and related proinflammatory cytokines. Parakeratosis, in which cell enucleation is inhibited and nuclei are retained within the stratum corneum, and melanocytes, also arises from ROS production and pro- 
A

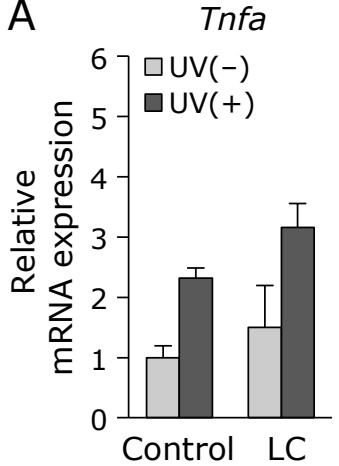

B

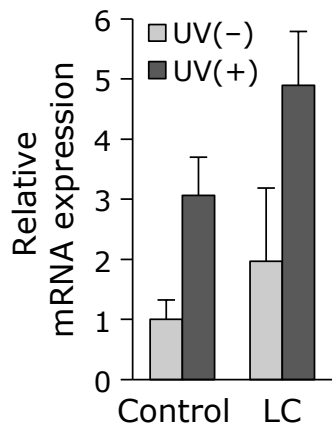

Fig. 5. Skin mRNA expression levels of pro-inflammatory cytokines. Skin mRNA expression normalized to the levels of $18 \mathrm{~S}$ ribosomal RNA as a loading control. The results of the two-way ANOVA were as follows: (A) LC diet: $p=0.048$, UVB irradiation: $p<0.001$; (B) LC diet: $p=$ 0.009 , UVB irradiation: $p<0.001$. Values represent the mean $\pm \operatorname{SE}(n=5)$.

inflammatory cytokine production following UVB irradiation. ${ }^{(21-23)}$

The mechanism underlying epidermal thickening as a result of LC diet intake, even without exposure to UVB radiation, has not yet been clarified. However, as pointed out in a previous study, this mechanism may be related to the following phenomena induced by LC diets: an increase in hepatic ROS levels, which is associated with a decrease in antioxidant enzymes, and subsequent translocation of ROS generated from the liver to skin via the blood vessels. ${ }^{(20)} \mathrm{LC}$ diets induce an increase in thiobarbituric acid reactive substances in the skin, promote chronic inflammation, and further suppress the autophagic activity associated with decreased mTOR activation. ${ }^{(11,24,25)}$ Therefore, ROS produced in the skin may amplify the ROS migrated from the liver to worsen skin morphology. In addition, skin damage resulting from LC diet intake while being exposed to UVB radiation appears to occur as a result of an increase in pro-inflammatory cytokines as well as NF- $\kappa \mathrm{B}$ and MMP activation, which may be directly induced by oxidative stress. ${ }^{(14,15,19)}$ This was demonstrated for the first time supported by our results, that the expression of the proinflammatory cytokines, TNF- $\alpha$ and IL- $1 \beta$, increases following ingestion of an LC diet in the skin. Therefore, similar to UVinduced photoaging, LC diets may increase damage under environmental factors known to adversely affect the epidermis, such as trace X-rays and urban particulate matter, ${ }^{(26,27)}$ leading to induction of the inflammatory response and subsequent skin damage. However, this study did not assess the effect of LC diets on MMP expression, which directly represents thickening of the epidermis, and proof of transfer of oxidative stress from the liver, thus, further research is needed to understand the mechanisms underlying these phenomena associated with LC diet intake, as well as to further clarify the effects induced by LC diets on skin morphology.

In non-obese mice, the administration of LC diets led to an increase in body weight and perirenal fat, however, did not affect blood glucose. It could be speculated that the hypertrophy of adipocytes in the LC diet triggered changes in adipocytokines and insulin sensitivity, which affect the blood glucose; however, our results showed no effects on blood glucose levels. Since insulin and leptin levels were not measured in this study, the mechanism underlying the control of blood glucose levels

\section{References}

1 Johnstone AM, Horgan GW, Murison SD, Bremner DM, Lobley GE. Effects of a high-protein ketogenic diet on hunger, appetite, and weight loss in obese men feeding ad libitum. Am J Clin Nutr 2008; 87: 44-55.

requires further study. In this experiment, UVB radiation reduced the number of adipocytes in both the subcutaneous and perirenal fat. With regard to the perirenal fat in particular, the adipocyte area, which was enlarged as a result of LC diet intake, decreased with UVB irradiation. This effect is probably related to the downregulation of Foxo1, which is known to inhibit adipocyte hypertrophy. The Foxol gene the pro-inflammatory cytokines IL-6, IL-8, and monocyte chemoattractant protein-3, produced by keratinocytes and fibroblasts by UVB irradiation, may be responsible for the reduction of subcutaneous fat. ${ }^{(28,29)}$

This is the first study, to our knowledge, to report that LC diets cause an increase in pro-inflammatory cytokines and morphological changes in skin, including epidermal thickening. Meanwhile, in the presence of UVB radiation, LC diets deteriorate the skin morphology by increasing the degree of parakeratosis and keratinization, while increasing the number of melanocytes and thickening the epidermis by decreasing the antioxidant capacity, processes that are known to result in an increase in the number of spots and wrinkles. Hence, LC diets may increase oxidative stress and pro-inflammatory cytokine production, thereby exacerbating the effects of UVB irradiation, which has a strong adverse effect on the body, particularly the skin.

\section{Author Contributions}

KK: acquisition of data, analysis of data, and writing-original draft preparation. TK: project administration, supervision, funding acquisition, and writing-review \& editing. RH: histological analysis, and technical advice. YO: acquisition of data.

\section{Acknowledgments}

We would like to thank M. Ichihashi, Y. Fukushima, and K. Sato for their valuable technical advice and discussion. We would also like to thank S. Yoshino for providing his expertise on the technicalities of UV irradiation. This work was supported by the Japan Society for the Promotion of Science Grant-in-Aid for Scientific Research (C) 18K11138 (to TK). We would like to thank Editage (www.editage.com) for their assistance with English language editing.

\section{Abbreviations \\ AP-1 activator protein-1 \\ Foxo1 forkhead box O1 \\ Gpx glutathione peroxidase \\ $\mathrm{H} \& \mathrm{E} \quad$ hematoxylin and eosin \\ LC low-carbohydrate \\ MMP matrix metalloproteinase \\ $\mathrm{NF}-\kappa \mathrm{B}$ nuclear factor- $\kappa \mathrm{B}$ \\ $\mathrm{PFC} \quad$ protein/fat/carbohydrate \\ ROS reactive oxygen species \\ SOD superoxide dismutase \\ UV ultraviolet \\ UVB ultraviolet B}

\section{Conflict of Interest}

No potential conflicts of interest were disclosed.

2 Noakes M, Keogh JB, Foster PR, Clifton PM. Effect of an energy-restricted, high-protein, low-fat diet relative to a conventional high-carbohydrate, lowfat diet on weight loss, body composition, nutritional status, and markers of 
cardiovascular health in obese women. Am J Clin Nutr 2005; 81: 1298-1306.

3 Meckling KA, Sherfey R. A randomized trial of a hypocaloric high-protein diet, with and without exercise, on weight loss, fitness, and markers of the Metabolic Syndrome in overweight and obese women. Appl Physiol Nutr Metab 2007; 32: 743-752.

4 Clifton PM, Bastiaans K, Keogh JB. High protein diets decrease total and abdominal fat and improve CVD risk profile in overweight and obese men and women with elevated triacylglycerol. Nutr Metab Cardiovasc Dis 2009; 19: $548-554$.

5 Layman DK, Boileau RA, Erickson DJ, et al. A reduced ratio of dietary carbohydrate to protein improves body composition and blood lipid profiles during weight loss in adult women. $J$ Nutr 2003; 133: 411-417.

6 Kaburagi T, Kanaki K, Otsuka Y, Hino R. Low-carbohydrate diet inhibits different advanced glycation end products in kidney depending on lipid composition but causes adverse morphological changes in a non-obese model mice. Nutrients 2019; 11: 2801.

7 Kadowaki S, Kaburagi T. Different effects on hepatic lipid metabolism of low carbohydrate diet on obese and non-obese mice. Diabetes Obes Int J 2017; 2: 000156.

8 Lagiou P, Sandin S, Lof M, Trichopoulos D, Adami HO, Weiderpass E. Low carbohydrate-high protein diet and incidence of cardiovascular diseases in Swedish women: prospective cohort study. BMJ 2012; 344: e4026.

$9 \mathrm{Hu}$ T, Mills KT, Yao L, et al. Effects of low-carbohydrate diets versus low-fat diets on metabolic risk factors: a meta-analysis of randomized controlled clinical trials. Am J Epidemiol 2012; 176 (Suppl 7): S44-S54.

10 He C, Wu Q, Hayashi N, Nakano F, Nakatsukasa E, Tsuduki T. Carbohydrate-restricted diet alters the gut microbiota, promotes senescence and shortens the life span in senescence-accelerated prone mice. J Nutr Biochem 2020; 78: 108326.

11 Okouchi R, Sakanoi Y, Tsuduki T. The effect of carbohydrate-restricted diets on the skin aging of mice. J Nutr Sci Vitaminol (Tokyo) 2019; 65 (Supplement): S67-S71.

12 Noto H, Goto A, Tsujimoto T, Noda M. Low-carbohydrate diets and all-cause mortality: a systematic review and meta-analysis of observational studies. PLoS One 2013; 8: e55030.

13 Ichihashi M, Ueda M, Budiyanto A, et al. UV-induced skin damage. Toxicology 2003; 189: 21-39.

14 Lephart ED. Skin aging and oxidative stress: Equol's anti-aging effects via biochemical and molecular mechanisms. Ageing Res Rev 2016; 31: 36-54.

15 Yamate Y, Hiramoto K, Sato EF. The preventive effect of coffee compounds on dermatitis and epidermal pigmentation after ultraviolet irradiation in mice. Skin Pharmacol Physiol 2017; 30: 24-35.
16 Kawaguchi Y, Tanaka H, Okada T, et al. The effects of ultraviolet A and reactive oxygen species on the mRNA expression of 72-kDa type IV collagenase and its tissue inhibitor in cultured human dermal fibroblasts. Arch Dermatol Res 1996; 288: 39-44.

17 Fisher GJ, Kang S, Varani J, et al. Mechanisms of photoaging and chronological skin aging. Arch Dermatol 2002; 138: 1462-1470.

18 Brenneisen P, Briviba K, Wlaschek M, Wenk J, Scharffetter-Kochanek K. Hydrogen peroxide $\left(\mathrm{H}_{2} \mathrm{O}_{2}\right)$ increases the steady-state mRNA levels of collagenase/MMP-1 in human dermal fibroblasts. Free Radic Biol Med 1997; 22: $515-524$

19 Klaunig JE, Kamendulis LM. The role of oxidative stress in carcinogenesis. Annu Rev Pharmacol Toxicol 2004; 44: 239-267.

20 Fisher GJ, Datta SC, Talwar HS, et al. Molecular basis of sun-induced premature skin ageing and retinoid antagonism. Nature 1996; 379: 335-339.

21 Naylor EC, Watson REB, Sherratt MJ. Molecular aspects of skin ageing. Maturitas 2011; 69: 249-256.

22 Ito M, Oguro K, Sato Y. Ultrastructural study of the skin in Sjögren-Larsson syndrome. Arch Dermatol Res 1991; 283: 141-148.

23 Halaban R, Langdon R, Birchall N, et al. Basic fibroblast growth factor from human keratinocytes is a natural mitogen for melanocytes. J Cell Biol 1988; 107: 1611-1619.

24 Kamada Y, Yoshino K, Kondo C, et al. Tor directly controls the Atg1 kinase complex to regulate autophagy. Mol Cell Biol 2010; 30: 1049-1058.

25 Solon-Biet SM, McMahon AC, Ballard JW, et al. The ratio of macronutrients, not caloric intake, dictates cardiometabolic health, aging, and longevity in ad libitum-fed mice. Cell Metab 2014; 19: 418-430.

26 Matsumoto KI, Ueno M, Nakanishi I, Indo HP, Majima HJ. Effects of lowdose X-ray irradiation on melanin-derived radicals in mouse hair and skin. $J$ Clin Biochem Nutr 2020; 67: 174-178.

27 Kitakaze T, Yoshioka Y, Furuyashiki T, Ashida H. Enzymatically synthesized glycogen protects inflammation induced by urban particulate matter in normal human epidermal keratinocyte. J Clin Biochem Nutr 2020; 67: 29-35.

28 Kitamura T, Ido Kitamura Y. Role of FoxO proteins in pancreatic beta cells. Endocr J 2007; 54: 507-515.

29 Kim EJ, Kim YK, Kim JE, et al. UV modulation of subcutaneous fat metabolism. J Invest Dermatol 2012; 131: 1720-1726.

This is an open access article distributed under the terms of the Creative Commons Attribution-NonCommercial-NoDerivatives License (http://creativecommons.org/licenses/by-nc-nd/4.0/). 